\title{
An Updated Account on Parents' Use of and Attitudes Towards Time-Out
}

\author{
Monica G. Canning ${ }^{1}$. Samantha Jugovac ${ }^{1} \cdot$ Dave S. Pasalich $^{1}$
}

Accepted: 11 September 2021 / Published online: 29 September 2021

(c) The Author(s), under exclusive licence to Springer Science+Business Media, LLC, part of Springer Nature 2021

\begin{abstract}
Time-out (TO) is a well-established technique for effectively managing childhood conduct problems, yet there is increasing controversy around its use. Using a mixed-methods approach, we investigated the impact of recent criticisms against TO on parents' use of and attitudes towards it. A community sample of parents $\left(N=297, M_{\text {age }}=37.14\right)$ completed an online survey (Study 1) to investigate use of and acceptability of TO. Results showed that $57.91 \%$ of parents reported using TO, slightly lower than past estimates. Perceived effectiveness and trust in clinicians predicted TO acceptability. To thoroughly examine negative attitudes towards TO, parents who did not use TO $\left(N=17, M_{\text {age }}=38.29\right)$ participated in individual interviews (Study 2). Amongst other findings, Study 2 found that parents view TO as punitive and contrary to their value of connection. We discuss implications for parenting interventions, dissemination of parenting information, and future research.
\end{abstract}

Keywords Parent management training $\cdot$ Parenting beliefs $\cdot$ Parent-child relationship $\cdot$ Discipline $\cdot$ Conduct problems

\section{Introduction}

Time-out (TO) is a key non-coercive discipline strategy used in parent management training (PMT) for the treatment of childhood conduct problems (CP). TO is based on principles of operant conditioning, and involves removing a child from a situation in response to $\mathrm{CP}$ and placing them in a less reinforcing setting where the caregiver does not actively engage with them [1]. TO is typically used with children aged 3-8 years to manage CP, both minor (e.g., mild episodes of noncompliance or temper tantrums) and more severe (e.g., physical violence) [1]. TO helps parents avoid inadvertent reinforcement of $\mathrm{CP}$ that occurs in coercive parent-child interactions [2], and was originally developed as an alternative to harsher strategies like smacking. TO, used in the context of a broader parenting intervention, may effectively reduce $\mathrm{CP}$, improve parent-child relationships, and prevent development of further problems for children [3]. In fact, TO is considered to be one of a few core strategies in PMT that help drive intervention effectiveness [4].

Dave S. Pasalich

dave.pasalich@anu.edu.au

1 Research School of Psychology, Australian National University, Canberra, ACT 2601, Australia

\section{Criticisms Towards TO}

Despite robust scientific consensus on positive outcomes associated with TO, there has been a recent explosion of criticisms. In their review on TO, Dadds and Tully [1] suggested this most recent spate of criticisms stemmed from a Time Magazine article in which Siegel and Bryson [5] equated TO with physical abuse, arguing that the brain of someone experiencing rejection (i.e., TO) looked like the brain of someone experiencing physical pain. However, no sound empirical evidence supported this claim with respect to TO specifically. A few months later, the authors retracted large parts of this article and clarified TO was not harmful when used properly [6]. Despite this retraction, TO's reputation may have been compromised and criticisms against TO have proliferated in the years since (e.g., [7]).

Of significance for shaping parents' negative perceptions of TO, these critical views are easily accessible across social media sites, parenting blogs, and books. On Time Magazine's Facebook page, liked by 12 million people, a post about Siegel and Bryson's article from 2015 has been shared more than two thousand times. By comparison, a post about restricting screen time for kids posted around the same time has only attracted 97 shares. This discrepancy suggests that, first, discussions surrounding the appropriateness of TO as a limit-setting technique have attracted great attention and, 
second, inaccurate information about TO has been shared far and wide. Dadds and Tully [1] reviewed internet-based criticisms about TO (e.g., $[5,7])$ and reported that, beyond TO being harmful, other major criticisms include that TO: damages the parent-child relationship, does not teach children any important skills, and fails to address underlying causes of CP. Crucially, these criticisms persist despite a complete lack of evidence that TO use is harmful in any way $[8,9]$.

\section{Consequences of Criticisms Towards TO}

Negative attitudes towards TO warrant further exploration. First, parents may use less empirically supported techniques if they choose not to use TO. A common alternative to TO suggested in most anti-TO communications is called time-in wherein parents physically stay with their children to help them manage their feelings following dysregulated or inappropriate behavior [10]. ${ }^{1}$ These sorts of parenting strategies have recently been coined 'exclusively positive parenting' [8], such that they do not include any disciplinary strategies at all. In general, the evidence base for exclusively positive parenting is lacking compared to interventions that include disciplinary components like TO [3, 8]. Moreover, from the perspective of operant conditioning theory, if time-in involves responding to children's $\mathrm{CP}$ with one-on-one parental attention, then this may positively reinforce negative child behaviors, such that a child may learn that by displaying a certain behavior, this will lead to their desired parent attention, making them more likely to repeat the behaviour in future. Hence, if parents decide not to use TO, they may employ alternative strategies with less empirical support as to their effectiveness and long-term outcomes for treating CP. In this light, there is a significant need to understand and target unwarranted negative attitudes towards TO.

Additionally, from an evidence-based practice (EBP) framework, it is critical that clinicians have an awareness of parents' attitudes towards parenting practices. Specifically, when working with families, either in a clinical capacity or at a population-health level, clinicians should use their clinical expertise while considering both TO's empirical basis and client-based factors, including parenting values, preferences, and beliefs about TO [11]. This implies a need to understand what these attitudes are to better tailor information and strategies. Although recent papers acknowledge and rebut criticisms against TO (e.g., [1,9]), these papers do not empirically examine parents' attitudes towards TO, nor mechanisms underlying these attitudes. We sought to address

\footnotetext{
1 This differs from "time-in" used in the context of TO, referring to reinforcing time spent with a parent that provides the contrast to the non-reinforcing TO environment.
}

these gaps in the literature to better understand the use of and possible issues with TO from parents' perspectives.

\section{Parents' Use of TO}

In addition to exploring parents' attitudes, updated knowledge on the number of parents currently using TO is needed, as recent articles suggest declining TO use (e.g., [1]). However, recent estimates show that TO remains a widely used strategy among parents in Western countries [12]. For instance, based on samples of US parents, Riley et al. [13] reported $76.8 \%$ of parents endorsed TO and Drayton et al. [14] reported $96 \%$ of their sample used TO. In the most recent estimate from an Australian sample, $78.5 \%$ of parents reported being likely or very likely to send a child to TO or "quiet-time" [15]. Thus, empirical estimates do not currently support the assumption of declining use.

However, these past studies have several limitations. Specifically, Drayton et al. [14] only asked participants to report if they have ever used TO, which does not take into account that some parents may not be currently implementing TO. Moreover, Sanders et al. [15] did not define either TO or "quiet time", therefore, it is not clear what these strategies looked like for parents who reported being likely to use them. An updated picture of current TO use is an integral first step in exploring parents' attitudes towards TO and will shed light on the prevalence of TO which, in turn, may reflect changing preferences towards TO. Additionally, limitations in previous studies suggest a need to provide a definition of TO to gain a more accurate estimate of TO use.

\section{Parents' Perceptions of the Acceptability of TO}

If TO is becoming less popular, the next significant question is why? A central factor is the extent to which parents find TO acceptable; that is, whether a strategy is appropriate for the problem at hand, reasonable, and fair [16]. Criticisms made against TO often state that it is not appropriate, reasonable, or fair. Thus, exposure to such criticisms is likely to influence parents' acceptability judgements. There are several variables that may underlie this influence. Each indicates a potential place in which efforts navigating negative attitudes could focus, and therefore, warrants investigation.

\section{Source of Information}

Given the differing views regarding TO acceptability between sources, where parents receive information is likely to influence their judgements of TO. In particular, participation in 'Web 2.0' environments may facilitate development of negative attitudes towards TO, whereas evidence-based information gleaned from scientific sources, including clinicians and journal articles, might foster positive attitudes. 
Web 2.0 represents a new era of online environments, characterised as interactive platforms with user-generated content (e.g., YouTube, blogs, Facebook; [17]). Web 2.0 environments are concerning in realms like healthcare because the credibility of shared information is unregulated. As such, these online environments can proliferate inaccurate information and initiate and maintain negative attitudes towards scientifically sound interventions. A well-researched example of this is evident in the anti-vaccination movement [17-19]. Anecdotal evidence exists of similar sorts of online groups (e.g., Facebook groups, parenting blogs) in the sphere of parenting philosophies and approaches, which share negative and inaccurate information about TO.

Conversely, it seems probable that evidence-based sources like clinicians and peer-reviewed journal articles should elicit positive attitudes towards TO acceptability, given the evidence supporting TO as an effective and appropriate limit-setting strategy. That being said, there is evidence that some clinicians advocate against the use of TO (e.g., $[5,7])$. Therefore, it is unclear exactly which sources of information (i.e. "non-scientific" sources like Facebook and parenting blogs or "scientific" sources like clinicians) are most likely to facilitate negative attitudes towards TO. This information is vital to understand how sources influence parents' attitudes.

\section{Trust in Clinicians}

Beyond the informational input itself, the credibility of a source also influences attitude formation. One central dimension of source credibility is trustworthiness [20]. Given that clinicians have an ethical responsibility to implement evidence-based strategies, and TO is well supported empirically, parents who question the trustworthiness of clinicians may find TO less acceptable. If this is the case, addressing negative attitudes towards TO may need to focus not only on providing accurate information to parents, but also on improving the perceived credibility of clinicians more broadly.

\section{Perceived Effectiveness}

Finally, a likely relevant part of the information parents receive about TO pertains to its effectiveness in reducing $\mathrm{CP}$; that is, doing what it is designed to do. Parents can receive information regarding effectiveness from external input or direct experience. A strategy is deemed acceptable when it is considered appropriate for a given problem [16], thus it is likely parents will tend to find TO acceptable if they believe it works to reduce CP. However, to the best of our knowledge this relationship has never been empirically tested. Increased clarity in this regard will help guide what it is about TO that turns some parents away.

\section{Changes in Parenting Paradigms and Values}

Finally, changing parental values regarding approaches to child-rearing may also underlie some negative attitudes parents hold towards TO. For instance, Coyne [21] suggested a paradigm shift amongst clinicians, with a general move from behavioral parenting approaches, including strategies like TO, to attachment-based approaches that predominantly focus on sensitively responding to children's needs underlying their behavior. If such a change is occurring, this would have considerable implications for dissemination and uptake of parenting programs. It would be particularly valuable to understand whether parents-who are consumers of parenting advice-hold similar views to those Coyne outlined amongst clinicians. Additionally, recent research suggests that parents who hold views contradictory to parenting science are not necessarily completely against science, but that the science they accept is guided by their pre-existing beliefs [22]. Therefore, understanding the values of parents is also important for more thoroughly exploring the complex interactions between parental values, trust in certain sources, and attitudes towards TO.

The current study aimed to gain insight related to the above considerations from a community sample of parents. Information about parents' values, attitudes towards, and understanding of $\mathrm{TO}$ in a general parenting population is highly valuable for clinicians. First, if clinicians do come across clients who are against the use of TO, having a deeper understanding of why this may be and how to mollify fears or offer appropriate alternatives, will improve outcomes for parents. Second, clinicians may engage with parents at a population-health level by writing parenting books or publishing parenting information on the internet. In this latter case, an understanding of the general views towards TO in the community is critical to effectively engaging with and educating parents.

\section{The Current Study}

This study aimed to address several significant gaps in the literature regarding parents' use of and attitudes towards TO. First, the literature is lacking a reliable and current estimate of TO use since the recent explosion of criticisms against it. An updated estimate is significant in determining reach and impact of criticisms. Second, if parents judge TO as an unacceptable strategy, it is important to understand what predicts these judgements so unwarranted critical attitudes can be more effectively navigated. Although TO is currently in the limelight in the parenting field, there is little research regarding parents' views on $\mathrm{TO}$ and the mechanisms underlying these attitudes. Finally, from the perspective of EBP, parents' values and beliefs must be appreciated rather than dismissed to deliver the 
best parenting services. Since parents' values and beliefs regarding TO are not yet known, this constitutes a real barrier to delivering appropriate and effective information to parents in both community and clinical settings.

We sought to address these gaps by employing a mixedmethods approach, combining online surveys with individual interviews with a community sample of parents. In Study 1, we administered an online survey to, first, estimate current usage of TO in Australian parents and, second, to explore whether acceptability of TO was influenced by: the source of parents' parenting information, the extent to which they trust clinicians' advice, and the extent to which they find TO effective. Regarding the second aim, we hypothesized that: despite evidence of some clinicians advocating against the use of TO, "scientific" sources should generally share scientifically accurate information about TO and, therefore, "non-scientific" sources of information would be associated with lower levels of acceptability compared to "scientific" sources (H1); parents who rated their trust in clinicians' advice highly would be more likely to find TO acceptable (H2); and perceptions of TO effectiveness would be positively associated with ratings of acceptability (H3).

To control for potentially confounding variables, we included measures of parental stress, parental self-efficacy, and parental gender as covariates. Participants completed this survey during national lockdown restrictions in response to COVID-19, in which many parents faced new challenges, such as home schooling. Therefore, increased parental stress may have affected participants' perspectives on discipline strategies and parenting in general. Parental self-efficacy refers to beliefs related to one's ability to parent [23] and is associated with persistence through parenting adversity [24]. Parental self-efficacy may influence study variables since parents who are more confident in their abilities may use different techniques and/or have different attitudes towards TO than those who are less confident. Finally, parental gender was included as a potential covariate given established parenting differences between mothers and fathers [25].

Study 2 aimed to use qualitative data from interviews with parents to corroborate and complement survey results, and to provide a rich and insightful look into parents' attitudes towards TO and potential mechanisms behind these attitudes.

\section{Study 1}

Using an online survey completed by parents, Study 1 aimed to provide an updated figure of TO use amongst parents of children aged 3 to 8 in Australia and explore predictors of TO acceptability.

\section{Method}

\section{Participants}

We recruited participants via Facebook ads, posts on popular parenting/general community pages on Facebook, online parenting forums, and emails to parents of children who attended after-school care centres. The Facebook pages/ forums were generally selected due to popularity of the page/forum (e.g., number of likes). Pages/forums that covered general parenting issues and advice were targeted (e.g., "Parenting Australia" Facebook page, "Bub Hub" forum), rather than those focusing on specific parenting approaches. However, given the general interest in TO amongst parenting circles, some included pages/forums had shared posts about TO in the past, both for and against the use of TO. In seeking a representative sample, we targeted Australian parents across different states. Eligible participants were 18 years and older, fluent in reading and writing English, residing in Australia, and the primary caregiver of at least one child aged 3 to 8 years.

There were 433 responses to the survey, though 135 of these were incomplete and not included in analysis due to lack of participant consent. Another participant was removed from analysis as a univariate outlier. A priori power analysis based on previous findings (e.g., [13]) indicated a necessary sample size of 164 to detect a small to medium effect size. Our final sample included 297 parents $(M$ age $=37.14$; $S D=5.61 ;$ range $=23-58$ years); 272 were female, 24 were male, and one identified as "other." Of this sample, 112 participants (37.7\%) reported that they had previously participated in some form of parent training.

Participants were from the eight states and territories in Australia, including 24.24\% from ACT, 20.20\% from NSW, $18.86 \%$ from Queensland, $15.49 \%$ from Victoria, $12.80 \%$ from South Australia, 7.07\% from Western Australia, 1.01\% from the Northern Territory, and $0.03 \%$ from Tasmania. Participants were representative of Australian parents in terms of age [26], and although ACT participants were overrepresented, the proportion of participants from other states and territories were similar to those in Australia at large [27]. Regarding ethnicity, 269 participants identified as Australian European, 16 as Asian, one each as Aboriginal and Torres Strait Islander, Hispanic, African, and Middle Eastern, and a further eight as an "other" ethnicity. Participants' highest levels of completed education were high school (6.06\%), certificate III/IV (9.09\%), diploma (19.87\%), bachelor's degree (48.48\%), master's degree (20.20\%), and $\mathrm{PhD}$ (5.39\%). Regarding average yearly household income, $20.87 \%$ of participants earnt less than $\$ 75,000,29.63 \%$ earnt between $\$ 75,000$ and $\$ 120,000$, and $49.50 \%$ earnt more than $\$ 120,000$. Most participants (98.65\%) were birth parents and $11.45 \%$ had children with a diagnosis of a mental health 
or developmental disability or disorder. Finally, $84.18 \%$ of participants were from two-caregiver households, $14.81 \%$ were from one-caregiver households, and $1.01 \%$ reported households with an "other" configuration.

\section{Procedure}

Participants completed the 10-15 min survey anonymously on the Qualtrics platform and were able to enter a raffle for one of two $\$ 75$ gift vouchers. Survey responses were collected between April and July 2020.

\section{Measures}

Given this study's novel research questions, we created a survey instrument using established and adapted measures.

Demographics Parents reported on their demographic information (e.g., gender, type of caregiver) described above.

TO Usage Parents were presented with a general description of TO borrowed from Riley et al. [13] ("When a child misbehaves, some parents will take the child out of that situation and have them go somewhere else. This is sometimes called "time-out". You may not call it that, but have you ever used time-out or something similar with your child?"). Parents indicated whether they used TO (ever, and if so, within the last month). These items had yes/no responses. If parents indicated they had never or did not currently use TO, they described strategies they used instead in a freeresponse form.

Source of Information Participants indicated where they received most of their information about $\mathrm{TO}$ from a list of potential sources. These included "scientific" (e.g., clinicians, peer-reviewed articles) and "non-scientific" (e.g., Facebook/other social media, friends and/or family) options. Participants could also choose "other" and specify the source in writing. We manually coded "other" responses as either "scientific" or "non-scientific". A score of 0 indicated a "non-scientific" source and a score of 1 indicated a "scientific" source.

Trust in Clinicians We created a 5-item scale to measure parents' trust in clinicians' advice (e.g., "I value the advice of clinicians over and above the advice of anyone else"). Items were adapted from the Trust in Physician Scale [28], which has demonstrated good construct and predictive validity, and high internal consistency (Cronbach's $\alpha=0.89$ ) [29]. Relevant items were chosen from the original 11-item scale and wording was adapted to refer to trust in clinicians regarding parenting advice. Participants responded on a Likert scale $(1=$ strongly disagree to $5=$ strongly agree $)$. Scores ranged from 5 to 25 , with higher scores indicating greater trust in clinicians. Cronbach's $\alpha$ in the current study was 0.90 .

Perceived Effectiveness of TO We created a 4-item scale to measure perceived effectiveness of TO. This scale was informed by the effectiveness subscale of the multidimensional adolescent satisfaction scale (MASS) (Cronbach's $\alpha=0.85$ ) [30] which asks adolescents to report their perceptions of an intervention having achieved a desired outcome (e.g., "The intervention helped my problems get better"). For Study 1 , items probed whether parents perceived that TO achieves its anticipated outcomes. Specifically, TO should decrease CP (e.g., "TO is effective in immediately decreasing inappropriate behaviors (e.g., hitting, ignoring instructions) in my child/ren"). Also, with proper implementation, children's behavior should improve such that the need for TO decreases over time [1] (e.g., "Time out is effective in decreasing inappropriate behaviors (e.g., hitting, ignoring instructions) in my child/ren in the long term"). Participants responded on a Likert scale $(1=$ strongly disagree to $5=$ strongly agree). Scores ranged from 4 to 20 , with higher scores indicating higher perceived effectiveness. Cronbach's $\alpha$ was 0.91 in our sample.

Parental Acceptability of TO We used a 6-item scale to measure participants' perceived TO acceptability. These items are from the acceptability subscale of the treatment evaluation inventory-short form (TEI-SF), which test parents' acceptability of limit setting strategies [31]. TEISF has good construct validity and internal consistency (Cronbach's $\alpha=0.85$ ) [31, 32]. Two non-relevant items were removed from the TEI-SF and remaining items were reworded to be more relevant to this study's aim (e.g., "Overall, I have a positive view towards time-out"). Participants responded to the items on a Likert scale $(1=$ strongly disagree to $5=$ strongly agree). Scores ranged from 6 to 30, with higher scores indicating greater acceptability. Cronbach's $\alpha$ for the current study was 0.97 .

Parental Stress Participants completed the 18 -item parental stress scale [PSS; 33] (e.g., "I sometimes worry whether I am doing enough for my child(ren)") (Cronbach's $\alpha=0.83$ ) [33] as a covariate measure. Participants responded to items on a 5-point Likert scale $(1=$ strongly disagree to $5=$ strongly agree). Nine items were reverse scored. Scores ranged from 18 to 90 , with higher scores indicating greater parental stress. Cronbach's $\alpha$ in the current study was 0.85 .

Parental Self-Efficacy Parental self-efficacy was examined as a potential covariate and measured using the 8-item parental self-efficacy sub-scale of the parenting sense of competence (PSOC) scale, which reflects parents' sense of competence in their role (e.g., "Being a parent is manage- 
Table 1 Descriptive statistics of continuous variables and spearman rank-order (or point biserial) correlations of main study variables in online survey

\begin{tabular}{|c|c|c|c|c|c|c|c|c|}
\hline & Range & M & $\mathrm{SD}$ & 1 & 2 & 3 & 4 & 5 \\
\hline 1. Source of information & $\mathrm{n} / \mathrm{a}$ & $\mathrm{n} / \mathrm{a}$ & $\mathrm{n} / \mathrm{a}$ & & & & & \\
\hline 2. Trust in clinicians & $5-24$ & 14.24 & 4.17 & -0.027 & & & & \\
\hline 3. Perceived effectiveness of TO & $4-20$ & 10.34 & 4.19 & -0.026 & $0.487 * *$ & & & \\
\hline 4. Acceptability of TO & $6-30$ & 16.10 & 7.06 & -0.013 & $0.558 * *$ & $0.870 * *$ & & \\
\hline 5. Parental self-efficacy & $8-42$ & 27.90 & 6.50 & 0.050 & $-0.217 * *$ & $-0.167 * *$ & $-0.192 * *$ & \\
\hline 6. Parental stress scale & $23-68$ & 41.79 & 8.30 & -0.018 & $0.171 * *$ & 0.092 & 0.087 & $-0.457 * *$ \\
\hline
\end{tabular}

$* p<0.05$

$* * p<0.01$

able, and any problems are easily solved") [34]. Participants responded on a Likert scale $(1=$ strongly disagree to $6=$ strongly agree). Scores ranged from 8 to 48 , with higher scores indicating higher self-efficacy. Cronbach's $\alpha$ in the current study was 0.86 .

\section{Analysis Plan}

To address Aim 1, we examined frequencies of responses related to TO use. To address Aim 2 (H1-H3), a hurdle model was applied due to inflation at the lower boundary of the acceptability variable [35]. A hurdle model comprises two steps. Firstly, we ran a binary logistic regression to explore factors predicting whether participants gave TO the lowest possible acceptability score. Secondly, we explored predictors' effects on the remainder of the distribution using multiple linear regression. We included potential covariates (i.e., parental stress and parental self-efficacy) and demographic variables (e.g., parental gender) in analyses when they were significantly associated with the dependent variable.

For qualitative data obtained from responses to "What do you use instead of TO?", the lead researcher made note of recurring responses. Recurring responses were refined into categories through discussion with all authors. All responses were coded into these categories. For this coding, and for coding of "other" responses in the source of information variable, we calculated an intercoder reliability (ICR) based on a sub-sample of responses coded by two authors. ICR values of $0.60-0.79,0.80-0.90$, and greater than 0.90 , indicated moderate, strong, and almost perfect agreement, respectively [36].

\section{Results and Discussion}

\section{Data Screening and Cleaning}

Scores for TO acceptability were inflated at their lower boundary value, so a hurdle model [35] was run to examine
Aim 2. All other major assumptions of multiple linear regression were met.

\section{Descriptive Statistics and Bivariate Correlations}

Table 1 presents descriptive statistics of all continuous variables and Spearman rank-order correlations between main study variables. TO acceptability showed significant positive associations with trust in clinicians and perceived effectiveness, and a significant negative association with parental self-efficacy. Trust in clinicians and perceived effectiveness were significantly positively associated.

\section{Frequency of TO Use}

For parents who said they had used TO, but not in the past month, their responses to "Why don't you currently use TO?", were coded as indicating that the participants (a) were TO "users" but had not needed it in the past month (e.g., "My son has given me no reason to use TO lately"), (b) had changed their attitude towards TO and were now "non-users" (e.g., "We have stopped using the TO system many years ago as we believe that it does not support our child's emotional wellbeing"), or (c) "other". The number of participants who reported they had used TO in the past month was added to those coded as TO "users", to obtain a total number of TO "users" $(N=172,57.91 \%)$. Similarly, the number of participants who reported they had never used TO was added to those coded as TO "non-users", to obtain a total number of TO "non-users" ( $N=120,40.40 \%)$.

If participants indicated they had never or were not currently using TO $(N=147)$, they were asked to describe parenting strategies they used instead. These responses were also coded into categories (kappa $=0.96, p<0.001,95 \%$ CI $[0.87,1.00]$ ) (see Table 2). Time-in or similar attachmentbased techniques were the most common response. This finding supports anecdotal evidence that parents replace TO with more permissive or non-behavioral (e.g., reasoning) techniques. 
Table 2 Frequency of responses to: "What sort of parenting strategies do you use instead of TO?"

\begin{tabular}{lll}
\hline Strategy & $\begin{array}{l}\text { Number of } \\
\text { responses }\end{array}$ & $\%$ of responses \\
\hline $\begin{array}{l}\text { Time-in or similar (e.g. connect- } \\
\text { ing, comforting, hugging) }\end{array}$ & 70 & 47.62 \\
$\begin{array}{l}\text { Talking (e.g. explaining, discuss- } \\
\text { ing, negotiating) }\end{array}$ & 68 & 46.26 \\
Consequences and rewards & 34 & \\
Emotion coaching & 19 & 23.13 \\
Distracting/redirecting & 13 & 12.93 \\
Role play/modelling & 8 & 8.84 \\
Voluntary quiet time & 5 & 5.44 \\
Other & 24 & 3.40 \\
\hline
\end{tabular}

Percentages add up to more than $100 \%$ because participants could specify more than one technique in their responses

Although the $57.91 \%$ of TO users in the current study is considerably lower than the estimate by Drayton et al. [14] of $96 \%$, this may be due to the present study also considering current TO use and reasons for not using TO. The current estimate was also lower than the most recent Australian estimate in which $78.5 \%$ of participants were likely or very likely to use TO or "quiet-time" [15]. Again, this may be because we provided a definition of TO in the survey, resulting in fewer participants reporting TO use when meaning something else. Overall, the addressing of limitations in these previous studies may partly account for the current lower estimate.

However, we employed a similar approach to Riley et al. [13] and still found a fall in TO use. We recorded fewer participants than Riley et al. having ever used TO $(72.05 \%$ vs. $76.8 \%)$ and having used TO in the last month $(50.51 \%$ vs. $57.52 \%$ ). Our reduced estimate when compared to a study using the same questions indicates that different approaches to questioning cannot fully explain the drop. Nevertheless, these differences are relatively small, and may be due to other differences between the current sample and Riley et al.'s sample, including the fact that the latter was a US sample. Thus, while the current study provides some support for a drop in TO use in recent years, further research is needed to more thoroughly explore changing TO use in Australian samples.

\section{Predictors of TO Acceptability}

Due to the inflated distribution of the acceptability variable, with 45 parents giving TO the lowest possible acceptability rating, a hurdle model examined predictors of parents' acceptability ratings of TO (i.e., Aim 2). A hurdle model involves first running a binary logistic regression to determine predictors of giving TO the lowest possible acceptability rating and, second, running a multiple linear regression on the remaining acceptability scores to explore predictors' effects on the rest of the distribution. We coded written responses given in the "other" category for the source of information variable as either "scientific" or "nonscientific" sources (kappa $=0.92, p<0.001,95 \%$ CI [0.73, 1.00]). Coding could not be determined for 16 participants where it was unclear whether the source should be labelled "scientific" or "non-scientific" (e.g., responses of "training course" and "day care/OSHC teacher"), and these participants were excluded from analyses involving the variable.

We ran a binary logistic regression to determine significant predictors of giving TO the lowest acceptability score. Acceptability was dummy coded, with participants giving the lowest possible acceptability score coded as $1(N=45)$ and all other participants coded as $0(N=236)$. Covariates of parental stress and parental self-efficacy were included in the model, as they were significantly associated with the dummy acceptability variable $(r=-0.18, p=0.002$ and $r=0.30$, $p<0.001$ respectively). The analysis was re-run excluding covariates and statistical significance remained the same. Overall, the model including covariates was significant, $\chi^{2}(5)=127.01, p<0.001$, and accounted for between 36.4 and $62.1 \%$ of variance.

Source of information was not a significant predictor of TO acceptability ( $\mathrm{B}=0.23, p=0.647$ ), so $\mathrm{H} 1$ was not supported. In support of $\mathrm{H} 2$ and $\mathrm{H} 3$, both trust in clinicians $(\mathrm{B}=-0.26, p<0.001)$ and perceived effectiveness $(\mathrm{B}=-0.44, p<0.001)$ were significant predictors. Examination of $\operatorname{Exp}(B)$ values showed that, for every unit increase in trust in clinicians and perceived effectiveness, the odds of a participant giving TO the lowest acceptability rating was decreased $0.77,95 \%$ CI $[0.67,0.89]$ and $0.64,95 \%$ CI $[0.53$, $0.76]$ times, respectively. Neither covariate reached significance in the model. Finally, no males in the sample gave TO the lowest acceptability rating. Therefore, the analysis was rerun on female participants alone. The same predictors reached significance, with similar $\operatorname{Exp}(\mathrm{B})$ values.

Next, a multiple regression analysis was employed to determine the effect of predictors on the rest of the distribution of scores for TO acceptability. The model was significant overall, $R^{2}=0.72, F(5,230)=119.60, p<0.001$. H1 was also not supported by this analysis, as source of information was not a significant predictor, $B=0.36, p=0.406,96 \%$ CI $[-0.49,1.21]$. Trust in clinicians predicted TO acceptability $B=0.24, p<0.001,95 \%$ CI [0.12, 0.37], supporting H2. Perceived effectiveness also predicted TO acceptability scores, $B=1.28, p<0.001,95 \%$ CI $[1.16,1.40]$, supporting H3. Again, neither covariate reached significance in this model. When covariates were removed from the model, the same predictors reached significance with similar B values.

Given significant gender differences in the acceptability variable, analysis was repeated for females only. The 
model remained significant, $F(5,206)=109.67, p<0.001$, $N=212$, and the same predictors reached significance as in the full model. For males only, the model remained significant overall, $F(5,18)=5.56, p=0.003, N=24$, however, only perceived effectiveness reached significance, $B=1.12$, $p<0.001,95 \%$ CI $[0.57,1.67]$. This is likely due to the small sample in this analysis, resulting in inadequate power.

Source of Information H1, that the source from which parents receive information would influence their acceptability judgements of TO, was not supported by analyses. Parents who received information from "scientific" sources (e.g., clinicians) were not more likely to find TO acceptable than parents who received information from "non-scientific" sources (e.g., Facebook). One explanation for this null result is that although an alignment between scientific sources and pro-TO information and non-scientific sources and anti-TO information was expected, there may have been a confound between source and information type. Indeed, as previously mentioned, some vocal advocates against TO are clinicians (e.g., $[5,7])$. Additionally, many pro-TO websites give inaccurate advice on how to use TO [37], meaning that parents who take this advice may implement TO incorrectly, reducing its effectiveness. Thus, there is evidence that sources coded as "scientific" may deliver inaccurate information regarding TO, not facilitating positive acceptability judgements. In a similar way, sources that we identified as "nonscientific" (e.g., "Facebook/other social media") may also not have included purely non-scientific information. For instance, some Facebook pages like those run by the government or health agencies may share scientifically accurate information about TO. In sum, the way "scientific" and "non-scientific" sources were classified in the current study may have missed some nuances surrounding the accuracy of information about TO within different sources. Future studies could ask participants to report specifically where or from whom they receive most of their information about TO to help reduce this confound.

Trust in Clinicians $\mathrm{H} 2$, that $\mathrm{TO}$ acceptability judgements would be positively associated with trust in clinicians, was supported by the results. Consistent with similar findings in the anti-vaccination literature (e.g., $[18,19])$, this link suggests negative attitudes towards TO may be associated with a broader mistrust of clinicians. However, support for $\mathrm{H} 2$ conflicts with the argument given above in response to the null result in $\mathrm{H} 1$, that some clinicians may not support TO, meaning trusting them would not necessarily facilitate positive attitudes towards the strategy. One potential explanation for these apparently conflicting findings is that parents may see the clinicians they receive anti-TO information from as somehow different from "mainstream" clinicians. Anecdotally, clinicians who are opposed to TO often present themselves in opposition to the mainstream and to "popular" techniques like TO (e.g., [5, 7]). Thus, it is possible that parents could simultaneously receive information from these clinicians who are opposed to TO and rate their trust in mainstream clinicians as low, with both predicting low acceptability judgements of TO, explaining the observed pattern in the current study.

If this is the case, an important question is why parents trust some clinicians but not others. One potential explanation is that some parents may have a general mistrust in "mainstream" clinicians or science. This is, as just noted, consistent with findings in the anti-vaccination literature [18]. Thus, clinicians positioning themselves as outside the mainstream may be viewed as more trustworthy by some parents. A second explanation is that parents trust clinicians who share their values. This would be consistent with a recent finding that parents accepted scientific findings when they were aligned with their pre-existing beliefs [22]. If there is, as Coyne [21] suggested, a paradigm shift in parenting from traditional behaviorism to attachmentbased approaches, this could explain why clinicians who are against behavioral techniques like TO may be more trusted by parents. Whatever the case, nuanced attitudes are clearly at play regarding relationships between information and source type, and parents' trust in clinicians. Study 2 explores the interplay between these variables in greater detail.

Perceived Effectiveness Finally, H3, that perceived effectiveness of TO would be positively associated with TO acceptability, was also supported by the results. However, two items in the acceptability scale (i.e., "I believe time-out is likely to be effective" and "I believe time-out is likely to result in permanent improvement") may have also measured perceived effectiveness, potentially accounting for the overlap. In a follow up analysis, we removed these items from the overall acceptability score and found that acceptability and perceived effectiveness remained highly correlated, $r_{s}=0.85, p<0.001$. This indicates the constructs were related conceptually, and not solely due to overlapping measurements. Put differently, believing TO does what it is supposed to do is associated with more positive attitudes towards TO, consistent with past literature [16]. However, given the interpretation above, that some parents may have negative attitudes towards TO because of a general mistrust of "mainstream" science or because TO runs counter to other pre-existing beliefs or values, it remains unclear whether simply giving parents more accurate information surrounding TO effectiveness is likely to improve attitudes. Rather, the current pattern of results seem to suggest that facilitating positive attitudes towards TO involves both providing parents with accurate information and ensuring parents are receptive to the sources from which accurate information comes. 
Overall, results from Study 1 simultaneously suggest that where parents receive information may not matter $(\mathrm{H} 1)$, but how much they trust certain sources does matter in predicting attitudes (H2). We have suggested a plausible explanation for this pattern of results, but more research is necessary to untangle the complex processes underlying parents' attitudes. Additionally, perceived effectiveness was a strong predictor of TO acceptability (H3), but it is unclear how effective improving access to information surrounding TO effectiveness will be without also addressing how receptive parents are to said information. Finally, the strong bivariate association between perceived acceptability and effectiveness of TO warrants additional investigation regarding the extent to which these variables reflect overlapping yet independent constructs. These ambiguities motivated Study 2 .

\section{Study 2}

In Study 2, we conducted qualitative interviews with parents who preferred not using TO with their children, to gain a deeper and more nuanced understanding of parents' attitudes towards TO, and to corroborate and expand on the quantitative findings in Study 1.

\section{Method}

\section{Participants}

Participant recruitment followed the same procedures outlined in Study 1, with the addition of asking participants to pass on the interview opportunity to other parents. Snowball sampling was appropriate for this qualitative investigation because participants themselves were best positioned to source other parents who would provide rich information [38]. The interviewer did not have pre-existing relationships/ affiliations with participants.

Seventeen parents (all birth parents) participated in interviews $(M$ age $=38.29 ; S D=5.21$, range $=31-48)$. We reached saturation with this sample size, meaning collection of more data was unlikely to shed any more light on parents' attitudes, indicated by little unique information emerging in later interviews [39]. Participant eligibility was the same as in Study 1 with the addition of participants nominating that they preferred not to use TO.

\section{Procedure}

Interviews were conducted individually with participants and audio-recorded on audio-only Zoom calls, lasting between 30 and $60 \mathrm{~min}$. Participants could enter a raffle for one of two $\$ 75$ vouchers as remuneration. All interviews were conducted from May-July 2020.

\section{Measures}

We designed a semi-structured interview for this study. Firstly, parents were asked: "Do you use time-out for managing your child/ren's inappropriate behavior? If not, why not?". Parents self-selected to participate in the interviews if they identified as preferring not to use TO with their children. As such, a specific definition of TO was not included because it was most meaningful for parents to voice their concerns about TO based on their own beliefs about what $\mathrm{TO}$ is. The remaining interview questions were about parents' views on TO, alternative techniques, parental values, and where they sought parenting information. Questions were open-ended to encourage in-depth responses and worded so as not to anticipate or provoke certain responses. There was also some idiosyncratic questioning, as is the nature of semi-structured interviews [40].

\section{Analysis Plan}

It is important for transparency that researchers are aware of potential bias and incorporate appropriate controls in qualitative research [39]. Two researchers involved in the study (D.P. and S.J.) had experience in delivering and evaluating both behavioral (e.g., PMT) and non-behavioral (e.g., attachment-based) parenting interventions. However, the lead researcher (M.C.), with no professional or parenting/ personal experience with $\mathrm{TO}$, collected all qualitative data. Bias was further reduced by discussing analyses and interpretations as a group, controlling undue influence of any individual interpretation [39].

Interview data was analysed using a hybrid approach of inductive and deductive thematic analysis for a thorough identification and exploration of themes [41]. That is, some codes were brought a priori to the data, based on past research and our research aims; however, other codes also emerged inductively from the data itself. Transcribed interviews were coded using NVivo 12. An initial coding by the lead researcher of a sub-set of interviews informed the creation of the first thematic map. This map underwent a collaborative iterative process of revision and modification, which ceased once all researchers agreed on the final themes. The lead researcher then coded all interviews. Codes covered between a single sentence up to a block of four or five sentences. To control for undue influence by the lead researcher on coding of the interviews, a second researcher coded a sub-set of the data with the view that analysis would only continue if a strong ICR $(0.80-0.90)$ was reached [36].

\section{Results and Discussion}

From thematic analysis of semi-structured interviews with 17 parents who preferred not to use TO, seven themes 


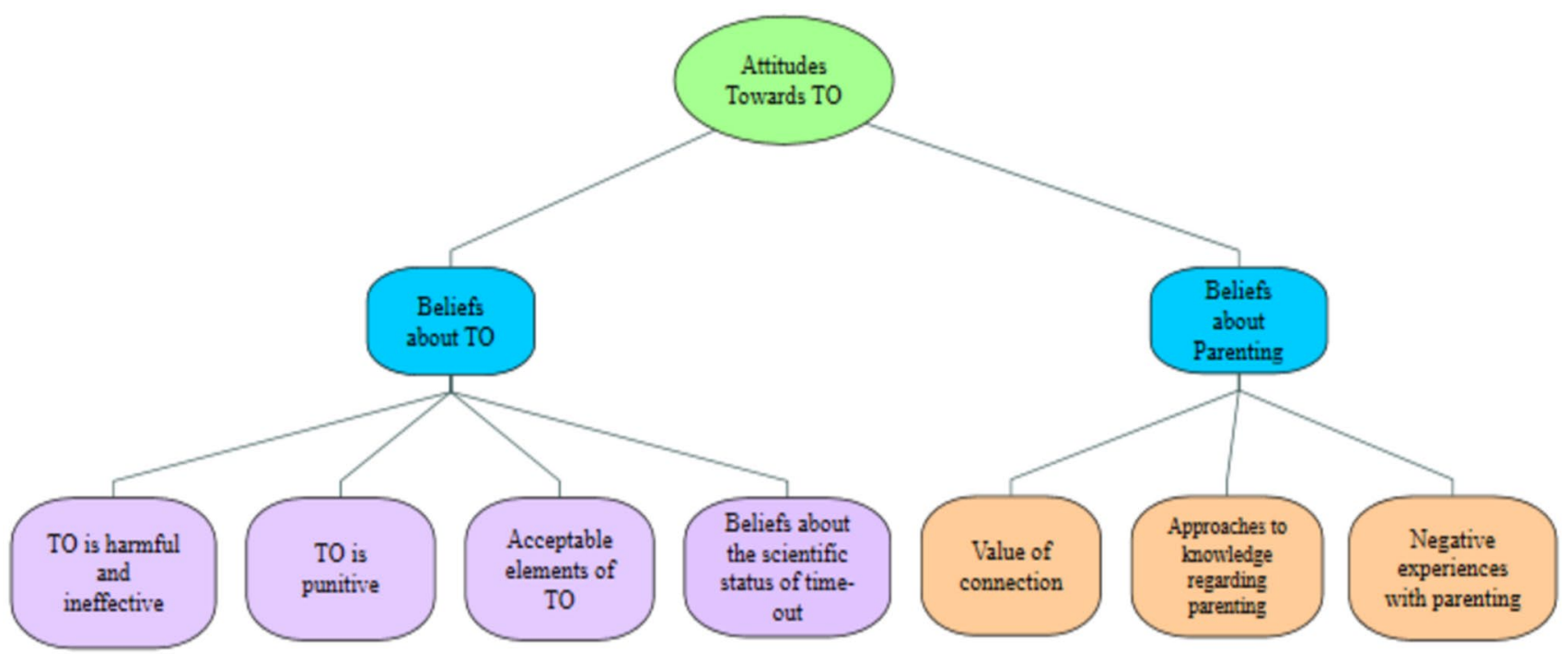

Fig. 1 Thematic map from analysis of parent interviews

emerged, sorted into two main categories (see Fig. 1). There was strong ICR between two raters on a subset of randomly selected excerpts (kappa $=0.82,95 \%$ CI $[0.71$, $0.90], p<0.001)$.

\section{Beliefs About TO}

TO is Harmful and Ineffective Over half of interviewed parents reported beliefs that TO was a harmful (52.94\%) and/ or ineffective strategy (70.59\%). Two main reasons given for these beliefs was that TO would not provide children with important life skills: "They're not really learning anything through this experience, in terms of how to self-regulate or how to help them deal with big emotions" (Participant 13) and TO did not deal with underlying causes of CP: "The reason they are misbehaving in the first place is because they have an unmet need, a physical or emotional unmet need" (Participant 2). This corroborated Study 1's findings that perceived effectiveness is important in predicting TO acceptability (H3). Additionally, these results highlighted beliefs that TO does not teach children important skills or deal with underlying causes of behavior as aspects of perceived effectiveness that were not considered in Study 1. Future studies should consider these elements of effectiveness to better understand how each interacts with acceptability.

TO is Punitive Most parents (88.24\%) described TO as a punishment, punitive, or an authoritarian strategy: "It's a little bit like jail but in a kid form" (Participant 11). This was broadly understood as a negative attribute, indicating that the word "punishment" holds negative connotations for some parents.
Acceptable Elements of TO Most parents (94.12\%) indicated that they thought some aspects of TO were acceptable. The most commonly endorsed aspect was removing children from situations: "I certainly use and am supportive of strategies where a child might be removed from a situation if they're displaying inappropriate behaviors" (Participant 19). In general, parents practising attachment-informed 'time-in' often endorsed removing a child from a situation, but not leaving them alone to self-regulate.

Beliefs About the Scientific Status of TO Some parents (23.53\%) believed science had proved TO was harmful or ineffective, and clinicians who recommended TO lagged behind: "[Official advice is] a bit slow to catch on to the newest, where the research is at, where the evidence is at" (Participant 16). It is unclear how parents developed this belief. However, one potential explanation for this finding is that inaccurate information about TO may have been given scientific credence by virtue of being shared by people who have scientific authority or by platforms with implied scientific credibility, such that parents came to believe that TO being harmful or ineffective was scientific fact.

\section{Beliefs About Parenting}

Approaches to Knowledge Regarding Parenting In response to if they trusted clinicians to give parenting advice, some parents (35.29\%) expressed beliefs that clinicians would give inaccurate or out-dated advice or would give advice bound by clinicians' own theoretical backgrounds or preferences: "[Clinicians] can believe whatever they want, they can be into more behavioral stuff or they can be into attachmentbased, it just depends on what they've learned and what they 
value" (Participant 2). This supports the link found in Study 1 between trust in clinicians and acceptability judgements of TO (H2), suggesting that some parents may have a general mistrust in mainstream clinicians which, in this case, translates as negative attitudes towards an evidence-based parenting technique.

Nevertheless, about half the sample (47.06\%) reported they would trust or take advice from various sources, including clinicians, if these sources aligned with the parent's pre-existing values: "It's less about trusting the source, like someone completely unqualified could tell me something that fits with my values and I'd be like, yes I want to take that on board" (Participant 5). These findings support the suggestion that some parents trust clinicians who are opposed to TO over mainstream clinicians due to an alignment of the parent's and the former clinician's values.

Value of Connection About half the sample (52.94\%) reported that they valued the connection, relationship, or attachment with their child/ren highly, often in terms of the threat TO posed to it: "TO, from my perspective, causes a sense of... it causes a disconnection and it restricts communication" (Participant 5). Additionally, this value appeared to drive the strategies parents used instead, with time-in or other attachment-based techniques being the most popular alternatives. This supports the anecdotal suggestions of a changing paradigm in parenting, with clinicians moving away from behavioral strategies like TO, to attachmentbased strategies [21], and further indicates a similar change taking place amongst parents.

Negative Experiences with Parenting Finally, some parents (58.82\%) referred to negative experiences in their own childhood as guiding choices they made with their own children: "I was raised in an environment with what I perceive to be a lot of arbitrary rules and I don't think that was constructive for my own development or my sense of belonging with family" (Participant 5). In other words, parents were avoiding TO because of the perceived authoritarian parenting they received, suggesting TO was seen as consistent with authoritarian or harsh parenting. Some parents (17.65\%) also had past negative experiences using TO with their children: "[My son] would get almost hysterical when he was put in the room by himself..." (Participant 13).

\section{General Discussion}

This research aimed to provide an updated estimate on the frequency of TO use in parents, investigate predictors of TO acceptability (H1-H3), and gain a rich insight into views and beliefs regarding TO in parents who prefer not to use TO. Findings from Study 1 show that, in our sample of 297
Australian parents, just over half are currently using TO. In Study 2, parents' reasons for not using TO echoed criticisms distilled by Dadds and Tully [1] from online sources [5, 7]. Specifically, parents believed that TO: (a) is harmful, (b) does not teach children important skills, (c) damages the parent-child relationship, and (d) does not get to the underlying cause of $\mathrm{CP}$. This provides partial support for the assumption that inaccurate information from online criticisms about TO has led to its rejection amongst some parents (e.g., [1]), and implies a need to address these criticisms. Moreover, we found that "scientific" sources like clinicians did not necessarily facilitate positive attitudes towards TO, and that some parents believed the criticisms made against TO by some clinicians were empirically supported, suggesting that inaccurate information about TO has somehow gained scientific credence. Furthermore, findings from both Study 1 and Study 2 suggest that parents who choose not to use TO are likely to use more permissive strategies like time-in. Given the relative scarcity of evidence regarding the effectiveness of parenting programs for child $\mathrm{CP}$ without disciplinary components $[3,8]$, these findings highlight the timely need to address negative attitudes towards TO.

Results from Study 1 demonstrate that where parents received information did not predict attitudes towards TO (H1), but how much they trusted certain sources of information did (H2). Study 2 showed that some parents did not trust clinicians for accurate parenting information at all. This is consistent with findings in the anti-TO literature that negative attitudes towards TO may be associated with a general mistrust in science and, in particular, "mainstream" science $[18,19]$. However, other parents in Study 2 were happy to trust clinicians if they shared their personal values or theoretical perspectives. These differences suggest parents may have different approaches towards the consumption of science, with some guided by a general anti-science view, and others guided by other values or beliefs which may take precedence over scientific findings. If this is the case, this would have significant implications for tailoring parenting information and support. For parents with a general antiscience viewpoint, efforts may need to be made at improving general trust in science. On the other hand, some parents may shun TO not because they are anti-science, but because they perceive it as inconsistent with their pre-existing values. Clinicians are likely to engage better with these parents when they are cognizant of and responsive to these values. We will discuss ways in which clinicians may be able to do so below.

Both Study 1 and Study 2 found that parents' perceptions of TO as effective positively predicted if they viewed it as acceptable (H3). Study 2 also indicated that some parents who chose not to use TO, believed it was ineffective in reducing $\mathrm{CP}$ in children, that it failed to teach children important skills (e.g., emotion regulation), and/or failed to 
address the underlying cause of CP. Thus, clinicians should emphasise TO's effectiveness in reducing $\mathrm{CP}$, as well as explain to parents how TO can improve important skills in children, including self-regulation of emotions and behavior [1]. However, overall results seem to suggest that facilitating positive attitudes towards TO involves not only providing parents with accurate information, but also ensuring parents are receptive to the sources from which accurate information comes.

Beyond corroborating and advancing quantitative findings from Study 1, additional themes and implications emerged from Study 2. For instance, Study 2 gave valuable insights into parents' beliefs about TO, important considerations for clinicians and researchers to engage effectively with parents in line with EBP [11]. Parents' sensitivity to words like "punishment", perceptions of TO as harmful or authoritarian, and information regarding which aspects of TO parents already find acceptable (i.e., removing a child from a situation), are very useful for guiding how TO should be framed. In particular, words like "punishment" could be avoided because of negative connotations they evoke. Also, clinicians could provide parents with theoretical background of why TO used properly, in the context of a warm parent-child relationship, is consistent with authoritative (not authoritarian) parenting [9], and how this type of parenting is associated with the best outcomes for children relative to other parenting styles [42]. Lastly, in line with accepted aspects of TO, forms of $\mathrm{TO}$ in which children are not left alone (non-exclusionary TO), could be a more acceptable way to present TO, compared to forms in which children are isolated. Overall, being aware of parents' conceptualizations means TO can be framed in ways consistent with pre-existing beliefs, potentially improving acceptance of the strategy.

Study 2 also uncovered the important value of connection for many parents. Additionally, several parents in Study 2 expressed aversion to TO based on past negative experiences in their own childhoods. For parents with strong child-rearing values that conflict with TO or with deep-seated beliefs stemming from childhood experiences, simply challenging their concerns about TO or presenting it in a different light may not be effective. Instead, there is a need for more research on the effectiveness of attachment-based strategies for responding to child $\mathrm{CP}$, to increase the availability of empirically-supported alternatives to TO for providing safe and consistent limit-setting that better align with the values of parents preferring not to use TO.

This information about parents' values, attitudes towards, and understanding of TO gleaned from a general parenting population is highly valuable for clinicians as it supports the notion of a shifting paradigm in parenting approaches [21]. The current study's results give clinicians a deeper understanding of why some clients may be against the use of TO. Moreover, when clinicians engage with parents at a population-health level (e.g., writing parenting books, publishing parenting information online), an understanding of general views towards TO in the community, as outlined above, is critical to effectively engaging with and educating these parents.

\section{Strengths and Limitations}

This research utilized a novel mixed-methods approach to thoroughly analyze parents' TO attitudes through integration of different data sources. Furthermore, we addressed a significant gap in the literature by examining multiple factors associated with parents' acceptability of TO. Notwithstanding these strengths, our study has important limitations. First, our sample was not representative of ethnic diversity in Australia, threatening generalizability of results. Given that endorsement of certain parenting strategies differ between ethnic groups [12], future research is needed to examine parents' attitudes towards TO, and discipline techniques more broadly, in samples of minority ethnic groups. Second, most parents in the survey sample reported their household income in the highest bracket. Given greater CP in low SES families [43], this suggests TO may not have been a necessary tool for some parents in the current sample. Third, mothers were overrepresented in the current study, threatening the generalizability of results to all parents.

Finally, we utilized a community, not a clinical, sample, so we cannot yet generalize to a clinical population. Nevertheless, as outlined above, information about values and attitudes towards TO from a general parenting sample is important to consider from a population-health perspective, meaning the current study still holds clinical relevance. Still, the need for TO may not have been as great amongst study participants compared to parents of children with more severe $\mathrm{CP}$ and the attitudes held by parents who seek treatment for their child/ren's CP may be different from those expressed by a community sample. That being said, the surveyed sample included a sizeable proportion $(37.7 \%)$ of parents who reported having previously participated in parent training. While it is not clear what type of parenting training (e.g., behavioral, attachment) these parents participated in, nor whether they were participating at the time of taking the survey, participation in parenting training was not significantly associated with use of or attitudes towards TO. This finding suggests that the attitudes towards TO uncovered in Study 1 may hold for both community and clinical samples; however, future studies should explore parents' use of and attitudes towards TO in a clinical sample and/or include a measure of CP severity, so these differences can be considered when interpreting results. 


\section{Summary}

The behavioral parenting strategy, TO, has decades of empirical support for its effectiveness and appropriateness in managing conduct problems in children. Yet, there has recently been an explosion of criticisms against its use. This study employed a novel mixed-methods approach to investigate the potential impact of these criticisms on parents' use of and attitudes towards TO, combining quantitative data from an online survey with qualitative data from interviews with parents. We found that TO use in our sample sits at around $57.91 \%$. Given the importance of early intervention for $\mathrm{CP}$, including population approaches to implementing PMT, and TO's robust empirical support for treating CP, insights gleaned from these studies have numerous implications for parenting intervention research and practice. For instance, emphasizing the empirical evidence regarding the effectiveness of TO and framing it to parents in ways consistent with pre-existing beliefs, may hold value in tailoring the implementation of TO for particular families. However, findings surrounding parents' approaches to knowledge, trust in clinicians, and parenting values also indicate a need to improve the empirical basis of alternative strategies to TO.

Acknowledgements This research was supported by an Australian Research Council Discovery Early Career Researcher Award (No. DE170100078) to Dave S. Pasalich.

\section{Declarations}

Conflict of interest The authors declare that they have no conflict of interest.

Ethical Approval All procedures performed in studies 1 and 2 were in accordance with the ethical standards of the Australian National University (Protocol 2020/088) and with the 1964 Helsinki declaration and its later amendments or comparable ethical standards.

Informed Consent Informed consent was obtained from all individual participants included in both studies.

\section{References}

1. Dadds MR, Tully LA (2019) What is it to discipline a child: what should it be? A reanalysis of time-out from the perspective of child mental health, attachment, and trauma. Am Psychol 74:794-808

2. Patterson GR, Reid JB, Dishion TJ (1992) Antisocial boys. Castalia Publishing Company, Eugene

3. Kaminski JW, Claussen AH (2017) Evidence base update for psychosocial treatments for disruptive behaviors in children. J Clin Child Adolesc Psychol 46:477-499

4. Kaminski JW, Valle LA, Filene JH, Boyle CL (2008) A metaanalytic review of components associated with parent training program effectiveness. J Abnorm Child Psychol 36:567-589
5. Siegel DJ, Bryson TP (2014) 'Time-outs' are hurting your child. Time Magazine. Accessed https://time.com/3404701/disci pline-time-out-is-not-good/

6. Siegel DJ, Bryson TP (2014) You said WHAT about time-outs?! Huffpost. Accessed https://rb.gy/dg47mm

7. Lapointe V (2016) 3 reasons why time-outs may be damaging your child. Time Magazine. Accessed https://time.com/4195647/ three-reasons-why-time-outs-may-be-damaging-your-child/

8. Larzelere RE, Gunnoe ML, Roberts MW, Lin H, Ferguson CJ (2020) Causal evidence for exclusively positive parenting and for timeout: rejoinder to holden, Grogan-Kaylor, Durrant, and Gershoff (2017). Marriage Fam Rev 56:287-319

9. Quetsch L, Wallace N, Herschell A, McNeil C (2015) Weighing in on the time-out controversy: an empirical perspective. Clin Psychol 68:4-19

10. Cooper G, Hoffman K, Powell B, Marvin R (2005) The circle of security intervention. In: Berlin L, Ziv Y, Amaya-Jackson L, Greenberg M (eds) Enhancing early attachments: theory, research, intervention and policy. Guilford Press, New York

11. Spring B (2007) Evidence-based practice in clinical psychology: what it is, why it matters; what you need to know. Clin Psychol 63:611-631

12. Prevoo MJ, Tamis-LeMonda CS (2017) Parenting and globalization in western countries: explaining differences in parent-child interactions. Curr Opin Psychol 15:33-39

13. Riley AR, Wagner DV, Tudor ME, Zuckerman KE, Freeman KA (2017) A survey of parents' perceptions and use of time-out compared to empirical evidence. Acad Pediatr 17:168-175

14. Drayton AK, Byrd MR, Albright JJ, Nelson EM, Andersen MN, Morris NK (2017) Deconstructing the time-out: what do mothers understand about a common disciplinary procedure? Child Fam Behav Ther 39:91-107

15. Sanders MR, Markie-Dadds C, Rinaldis M, Firman D, Baig N (2007) Using household survey data to inform policy decisions regarding the delivery of evidence-based parenting interventions. Child Care Health Dev 33:768-783

16. Kazdin AE (1980) Acceptability of alternative treatments for deviant child behavior. J Appl Behav Anal 13:259-273

17. Kata A (2012) Anti-vaccine activists, web 2.0, and the postmodern paradigm - an overview of tactics and tropes used online by the anti-vaccination movement. Vaccine 30:3778-3789

18. Davies P, Chapman S, Leask J (2002) Antivaccination activists on the world wide web. Arch Dis Child 87:22-25

19. Smith N, Graham T (2019) Mapping the anti-vaccination movement on Facebook. Inf Commun Soc 22:1310-1327

20. Ma TJ, Atkin D (2017) User generated content and credibility evaluation of online health information: a meta analytic study. Telemat Inform 34:472-486

21. Coyne J (2013) Parenting from the outside-in: reflections on parent training during a potential paradigm shift. Aust Psychol 48:379-387

22. Scott JK, Gershoff ET (2020) Trust in expert versus lay comments in online articles about spanking and car seat safety. J Fam Psychol 35:399-409

23. Jones TL, Prinz RJ (2005) Potential roles of parental self-efficacy in parent and child adjustment: a review. Clin Psychol Rev 25:341-363

24. Bandura A (1977) Self-efficacy: toward a unifying theory of behavioral change. Psychol Rev 84:191-215

25. Möller EL, Majdandžić M, de Vente W, Bögels SM (2013) The evolutionary basis of sex differences in parenting and its relationship with child anxiety in Western societies. J Exp Psychopathol 4:88-117

26. Australian Bureau of Statistics (2011) Median age of parents. Accessed https://www.abs.gov.au/ausstats/abs@.nsf/Products/ EB94F978573DD478CA25793300167581 
27. Australian Bureau of Statistics (2020) National, state and territory population. Accessed https://www.abs.gov.au/statistics/people/ population/national-state-and-territory-population/latest-release

28. Anderson LA, Dedrick RF (1990) Development of the trust in physician scale: a measure to assess interpersonal trust in patientphysician relationships. Psychol Rep 67:S1091-1100

29. Thom DH, Ribisl KM, Stewart AL, Luke DA, The Stanford Trust Study Physicians (1999) Further validation and reliability testing of the trust in physician scale. Med Care 37:510-517

30. Garland AF, Saltzman MD, Aarons GA (2000) Adolescent satisfaction with mental health services: development of a multidimensional scale. Eval Program Plann 23:165-175

31. Kelley ML, Heffer RW, Gresham FM, Elliott SN (1989) Development of a modified treatment evaluation inventory. J Psychopathol Behav Assess 11:235-247

32. Adams CD, Kelley ML (1992) Managing sibling aggression: overcorrection as an alternative to time-out. Behav Ther 23:707-717

33. Berry JO, Jones WH (1995) The parental stress scale: Initial psychometric evidence. J Soc Pers Relat 12:463-472

34. Johnston C, Mash EJ (1989) A measure of parenting satisfaction and efficacy. J Clin Child Psychol 18:167-175

35. Cragg JG (1971) Some statistical models for limited dependent variables with application to the demand for durable goods. Econometrica 39:829-844

36. McHugh ML (2012) Interrater reliability: the kappa statistic. Biochem Med 22:276-282
37. Corralejo SM, Jensen SA, Greathouse AD, Ward LE (2018) Parameters of time-out: research update and comparison to parenting programs, books, and online recommendations. Behav Ther 49:99-112

38. Patton MQ (2002) Qualitative research \& evaluation methods: Integrating theory and practice. SAGE Publications, California

39. Boeije HR (2009) Analysis in qualitative research. SAGE Publications, London

40. Longhurst $\mathrm{R}$ (2016) Semi-structured interviews and focus groups. In: Clifford N, Cope M, Gillespie T, French S (eds) Key methods in geography. Sage, London

41. Fereday J, Muir-Cochrane E (2006) Demonstrating rigor using thematic analysis: a hybrid approach of inductive and deductive coding and theme development. Int J Qual Methods 5:80-92

42. Baumrind D (1966) Effects of authoritative parental control on child behavior. Child Dev 37:887-907

43. Dodge KA, Pettit GS, Bates JE (1994) Socialization mediators of the relation between socioeconomic status and child conduct problems. Child Dev 65:649-665

Publisher's Note Springer Nature remains neutral with regard to jurisdictional claims in published maps and institutional affiliations. 